\title{
Structural and Vibrational Characterization of Tetracyanoethylene-Hexamethylbenzene as a Function of Pressure
}

\author{
Jennifer A. Ciezak *,+* and Juscelino B. Leão ${ }^{\dagger}$ \\ NIST Center for Neutron Research, National Institute of Standards and Technology, Gaithersburg, \\ Maryland 20899, and U.S. Army Research Laboratory, Weapons and Materials Research Directorate, \\ Aberdeen Proving Ground, Maryland 21005
}

Received: November 3, 2005; In Final Form: January 17, 2006

\begin{abstract}
The neutron powder diffraction and inelastic neutron scattering (INS) spectra of the electron donor-acceptor complex, tetracyanoethylene-hexamethylbenzene have been studied as a function of pressure to $0.414 \mathrm{GPa}$. Using the PW91 and PBE density functional theories, the unit cell vectors were calculated as a function of pressure and are compared to those experimentally obtained from the diffraction data. The calculated lattice vectors display large errors at low pressures but were found to be in close agreement with the experimental vectors at $0.414 \mathrm{GPa}$. Comparison of the experimental INS spectra of the TCNE-HMB enabled assignment of specific vibrational modes while providing a direct measurement of the effect of pressure on the complex. The PW91 vibrational frequency calculations reproduced both the vibrational intensities and frequencies with relative accuracy.
\end{abstract}

\section{Introduction}

The existence of several novel and unique physical properties of electron donor-acceptor complexes, such as magnetism, ${ }^{1}$ conductivity, and superconductivity, ${ }^{2}$ has generated considerable interest in recent years. It has been discovered that through control of external parameters such as temperature, pressure, and magnetic field strength, the magnitude of these properties can be tuned. ${ }^{3}$ Although these materials are rapidly gaining extensive employment as integrated circuit components and sensors for mobile phones, ${ }^{4}$ widespread potential exists for other applications. These complexes have been experimentally characterized for many years, yet much of the chemistry and physics of these materials that result in such unusual properties is just now being discovered. For new applications to emerge, it is necessary to first gain a deeper understanding of the fundamental characteristics of these materials, such as the relationship between intramolecular and intermolecular interactions, the internal conformation, relative orientation, and vibrational behavior.

Electron donor-acceptor (EDA) complexes are typically weakly interacting compounds that are formed when a donor molecule, $D$, donates an electron to an acceptor molecule, $A .^{3}$ The bonding arrangement in the solid-state is governed by electrostatic forces, and in most cases, the donor and acceptor molecules are arranged in stacks consisting of alternating donor and acceptor ions. The stacks are held together by weakly interacting van der Waals forces. The open-shell character of EDA complexes commonly results in electron-electron, electron-phonon, and spin coupling in the solid-state, as well as a variety of electronic instabilities, such as Mott, Peierls, and spin-Peierls transitions. ${ }^{5}$ Although vast experimental knowledge

* Corresponding author. Address: 100 Bureau Dr., MS 8562, Gaithersburg, MD 20899. Tel: (301) 975-6082. Fax: (301) 921-9847. E-mail: jciezak@arl.army.mil.

National Institute of Standards and Technology.

$\doteqdot$ U.S. Army Research Laboratory, Weapons and Materials Research Directorate. about EDA complexes exists, theoretical descriptions remain rare and it is only in recent years several papers have appeared on the subject. ${ }^{6-11}$

Solid-state quantum chemical calculations are routinely used to examine the properties of solids, interfaces, and surfaces for a wide range of material classes and offer new hope in solving difficult quantum chemical problems that require more parameters than can be included in an isolated molecule simulation. By using a combined theoretical/experimental approach, the accuracy of these theoretical methods has greatly improved in recent years. Inelastic neutron scattering (INS) spectroscopy is a vibrational spectroscopic technique commonly employed as a fundamental test of theoretical methods. Inelastic neutron scattering spectra can be directly correlated to the normal mode eigenvectors, which are part of the standard output of an ab initio simulation. The large scattering cross-section of hydrogen can be exploited to obtain a vibrational spectrum that reveals primarily hydrogen motion. The intensity of all lattice and molecular vibrations observed in the spectrum is directly proportional to the sum of the squares of the hydrogen displacement vectors for a particular normal mode of vibration. Numerous recent investigations comparing INS vibrational and theoretical spectra exist, including a recent report on an EDA complex formed between tetracyanoquinodimethane (TCNQ) and hexamethylbenzene. ${ }^{11-19}$

The focus of this work concerns the electron donor-acceptor complex formed between tetracyanoethylene and hexamethylbenzene (TCNE-HMB), shown in Figure 1. At room temperature, X-ray diffraction shows equally spaced TCNE and HMB molecules arranged in a DADADA arrangement with an intermolecular spacing of $3.35 \AA{ }^{20}$ This spacing decreases to $3.28 \AA$ at $113 \mathrm{~K}^{21}$ The TCNE-HMB complex has been subject to extensive experimental characterization using X-ray diffraction, ${ }^{20,21}$ optical absorption spectroscopy, ${ }^{22-24}$ infrared spectroscopy, ${ }^{25-30}$ Raman scattering ${ }^{31-37}$ and most recently, inelastic neutron scattering. ${ }^{10}$ Several attempts have been made to obtain an accurate theoretical representation of the complex, but many 

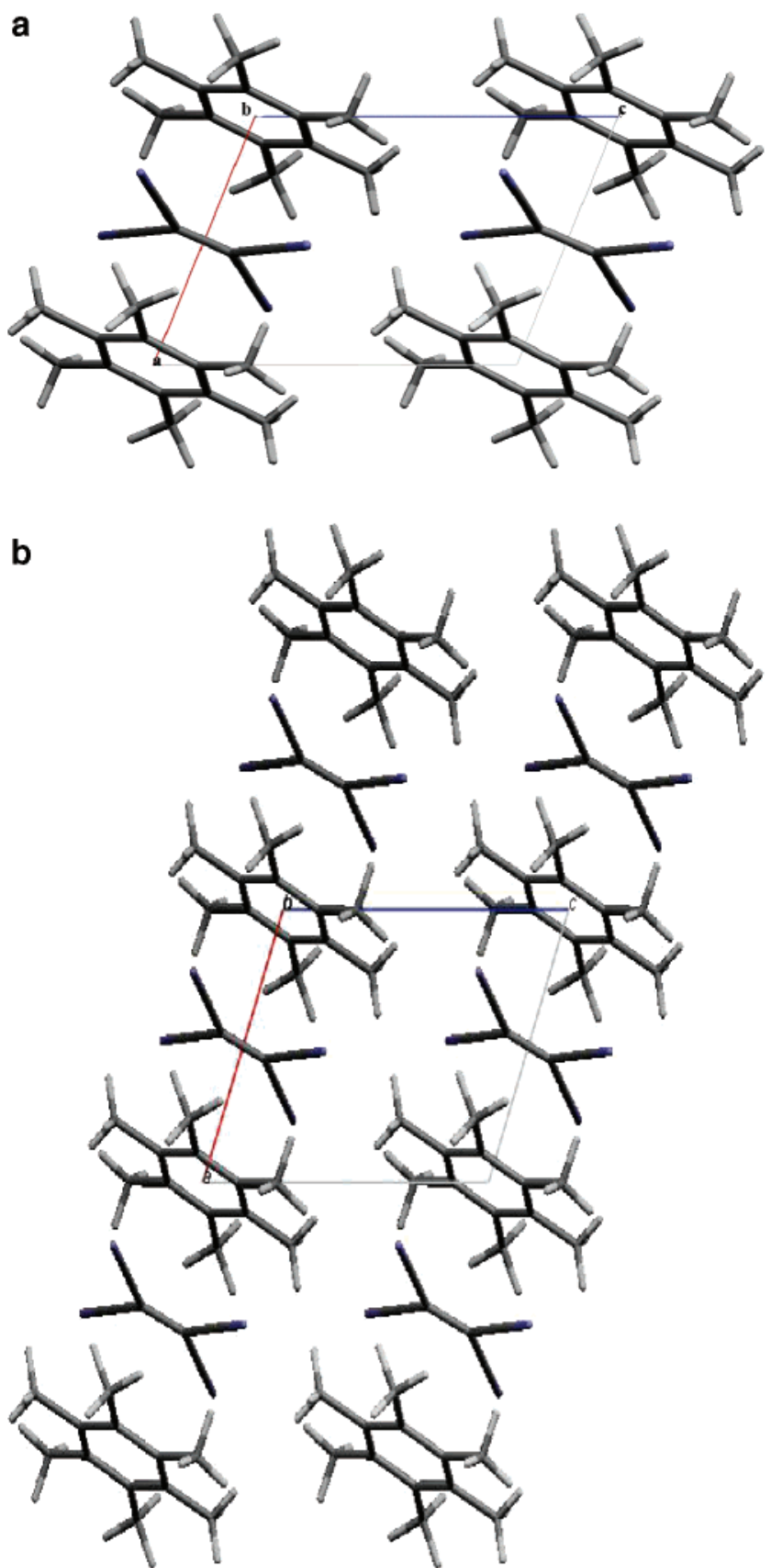

Figure 1. Molecular geometry of the TCNE-HMB complex shown (a) in the unit cell and (b) as an expanded view showing the stacking arrangement.

of these studies have been limited due to use of an isolated complex as the model for the simulations., ${ }^{9,28,38}$ Recent solidstate calculations have demonstrated the importance of including the intermolecular interactions to obtain an adequate description of the TCNE-HMB complex. ${ }^{10}$

Optical absorption ${ }^{39-44}$ and infrared spectroscopy ${ }^{44,45}$ have been used to characterize pressure-induced changes of the TCNE-HMB complex in solution and the solid-state, respectively. Optical spectra obtained as a function of pressure indicated an increase in the ionic character of TCNEHMB. ${ }^{39-44}$ This is corroborated with the IR data that showed all vibrational frequencies linearly increase as a function of pressure ${ }^{44,45}$ Interestingly, the intensity of the $\mathrm{C}=\mathrm{C}\left(\mathrm{A}_{\mathrm{g}}\right)$ band of TCNE increased almost 5 times between 0 and $10 \mathrm{GPa}$. The increase in intensity as a function of pressure for this particular vibration was proposed to be due to a subtle shift of the TCNE molecule from the site symmetry occupied in the atmospheric pressure crystal structure. ${ }^{45}$ This shift was expected to be at, or near, the resolution limit of the X-ray diffraction structure; thus no crystallographic analysis was undertaken at that time. As such, it seems justified to seek an understanding of the pressure dependent physical properties of the TCNE-HMB complex.

We report here the results of neutron diffraction, inelastic neutron scattering, and theoretical studies of TCNE-HMB compressed to $0.414 \mathrm{GPa}$ at $50 \mathrm{~K}$. Our neutron diffraction studies to $0.414 \mathrm{GPa}$ show a much higher compression of the $b$ axis, relative to the $a$ and $c$ axes. Large errors are observed in the calculated unit cell vectors, which systematically decrease as the pressure increases. Presumably, these errors in the unit cell vectors reflect the lack of van der Waals' forces implemented in current density functional theory (DFT) functionals. This phenomenon has also been reported during crystal structure predictions of energetic materials. ${ }^{46}$ Vibrational modes for the solid-state TCNE-HMB complex under pressure were generated using density functional theory ab initio quantum mechanical calculations. Good correspondence between the calculated modes and experimentally obtained spectra allowed the assignment of vibrational modes to the experimentally observed INS peaks.

\section{Materials and Methods}

Experimental Details. Tetracyanoethylene and hexamethylbenzene were obtained from Sigma Aldrich and used without further purification. The EDA complex, TCNE-HMB, was synthesized according to previously published methods. ${ }^{21}$ Approximately $1.5 \mathrm{~g}$ of the TCNE-HMB complex was ground into a fine powder and loaded into a cylindrical aluminum pressure cell and placed into a top-loading cryostat. The polycrystalline powder was held at $50 \mathrm{~K}$ for the duration of the experiments. The pressure cell used for these experiments is constructed from a $40 \mathrm{~mm}$ diameter cylindrical piece of aluminum and is able to reach a maximum pressure of 0.414 $\mathrm{GPa}$. The sample compartment is a cylindrical opening $5 \mathrm{~mm}$ in diameter and $45 \mathrm{~mm}$ long. One end of the cell is sealed using a steel ball and screw mechanism and the other end is connected to the pressure intensifier by a stainless steel capillary. Specific details concerning the method for pressurizing the cell can be found at the NIST Center for Neutron Research website. ${ }^{47}$

Neutron Powder Diffraction. Angle-dispersive neutron powder diffraction experiments, using a neutron wavelength of $1.5402 \AA$, were carried out at the BT-1 thirty-two-detector neutron powder diffractometer at the National Institute of Standards and Technology Center for Neutron Research (NCNR) in Gaithersburg, MD. The $\mathrm{Cu}$ (311) monochromator was employed for all of the experiments described herein and data were collected over the range $3-168^{\circ} 2 \theta$ for approximately 24 $\mathrm{h}$ at each pressure. ${ }^{48}$ The General Structure Analysis program ${ }^{49}$ graphical user interface EXPGUI ${ }^{50}$ employing Rietveld refinement was used to obtain the unit cell parameters in the $5-36^{\circ}$ $2 \theta$ and $46-165^{\circ} 2 \theta$ ranges. Diffraction between $36^{\circ}$ and $46^{\circ}$ $2 \theta$ is dominated by two intense aluminum diffraction peaks, which represent the (001) and (111) crystal planes of the pressure cell. The parameters used for the initial refinement of the TCNE-HMB complex at atmospheric pressure and $50 \mathrm{~K}$ were obtained from the Cambridge Structural Database and are as follows: space group $=P \overline{1}, a=6.563 \AA, b=8.614 \AA, c=$ $8.656 \AA, \alpha=108.22^{\circ}, \beta=102.61^{\circ}, \gamma=111.74^{\circ}, Z=1$. At all other pressures, the initial refinement parameters were the final results obtained in refining the diffraction pattern of the next lower pressure. 
Inelastic Neutron Scattering. Inelastic neutron scattering spectra were collected between 40 and $1200 \mathrm{~cm}^{-1}$, on the Filter Analyzer Neutron Spectrometer (FANS) located at BT-4 at the NIST Center for Neutron Research, through the use of both the copper $(\mathrm{Cu}(220))$ and pyrolytic graphite (PG (002)) monochromators. The scattered neutrons were collimated with 20/ $20 \mathrm{~min}$ of arc divergence prior to inelastic scattering by the sample. The scattered neutrons first passed through a bismuth filter that removes any spurious Be-phonon excitations and then Bragg cutoff filters of polycrystalline beryllium and graphite that remove all neutrons with energy greater than $1.8 \mathrm{meV}$. Though the FANS instrument is capable of scanning from $\sim 32$ to $1700 \mathrm{~cm}^{-1}$, this experiment focuses only on the $40-1200$ $\mathrm{cm}^{-1}$ range. More specific details are available regarding the FANS instrument. ${ }^{51}$ All experimental INS spectra presented in this paper were normalized for background scattering using the Data Analysis and Visualization Environment (DAVE) program..$^{52}$

Computational Details. The calculated crystal parameters and normal modes of vibrations were obtained as a function of pressure using the Vienna Ab Initio Simulation Package (VASP). ${ }^{53}$ The lattice parameters were obtained as a function of pressure using both the Perdew-Burke-Ernzerhof $(\mathrm{PBE})^{54}$ and Perdew-Wang 91 (PW91) $^{55}$ functionals. Only the vibrational results using the PW91 functional are presented here, as we were unable to obtain reliable vibrational frequencies using the PBE functional. Vanderbilt ultrasoft pseudopotentials (USP) ${ }^{56}$ and Monkhorst-Pack $k$-point generation methods were used for all calculations. Calculations were run at 280, 330, 430, 495, 545 , and $645 \mathrm{eV}$ to test for convergence, which was noted at $545 \mathrm{eV}$.

In all calculations, no symmetry restrictions were imposed. At atmospheric pressure, the calculations were started from the unit cell and structural parameters listed in the Cambridge Structural Database. ${ }^{21}$ The electronic energies were allowed to converge to $2.0 \times 10^{-6} \mathrm{eV}$ and the structure was considered converged once the difference in free energy between gradient steps was less than $2.0 \times 10^{-5} \mathrm{eV}$. Calculations were done at $0.138,0.276$, and $0.414 \mathrm{GPa}$ to coincide with our experimental measurements. These calculations were started from the optimized structures obtained from optimizing the structure of the next lowest pressure. The optimized molecular geometries were then used to determine the Hessian matrix and the vibrational frequencies of the TCNE-HMB complex via the finite difference method. The theoretical inelastic neutron scattering spectra were constructed from the normal mode eigenvectors using the A-Climax program v.5.1.3. ${ }^{57,58}$

\section{Results and Discussion}

Unit Cell. Neutron diffraction patterns were obtained for the TCNE-HMB complex as a function of hydrostatic pressure to $0.414 \mathrm{GPa}$. Because only the first twenty peaks of the experimental data were available for refinement, it was not possible to assign the atomic coordinates to each molecule in the asymmetric unit cell using Rietveld analysis. Therefore, we will not discuss the accuracies of the calculated molecular geometries. Instead, this section will focus on the accuracy of the calculated unit cell parameters as a function of pressure.

The experimental unit cell parameters are reported in Table 1 as a function of pressure. The unit cell parameters and volume obtained at $50 \mathrm{~K}(0 \mathrm{GPa})$ are similar to the parameters and volume reported at $113 \mathrm{~K} .{ }^{21}$ The volume decreases by $0.24 \%$ upon cooling from 113 to $50 \mathrm{~K}$. As expected, the overall experimental volume of the unit cell decreased with increasing pressure from $398.626 \AA^{3}$ at $50 \mathrm{~K}(0 \mathrm{GPa})$ to $377.221 \AA^{3}$ at

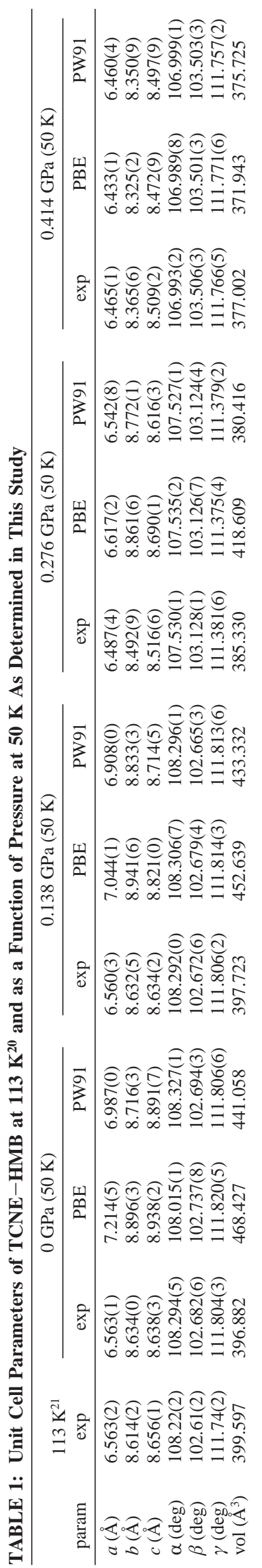



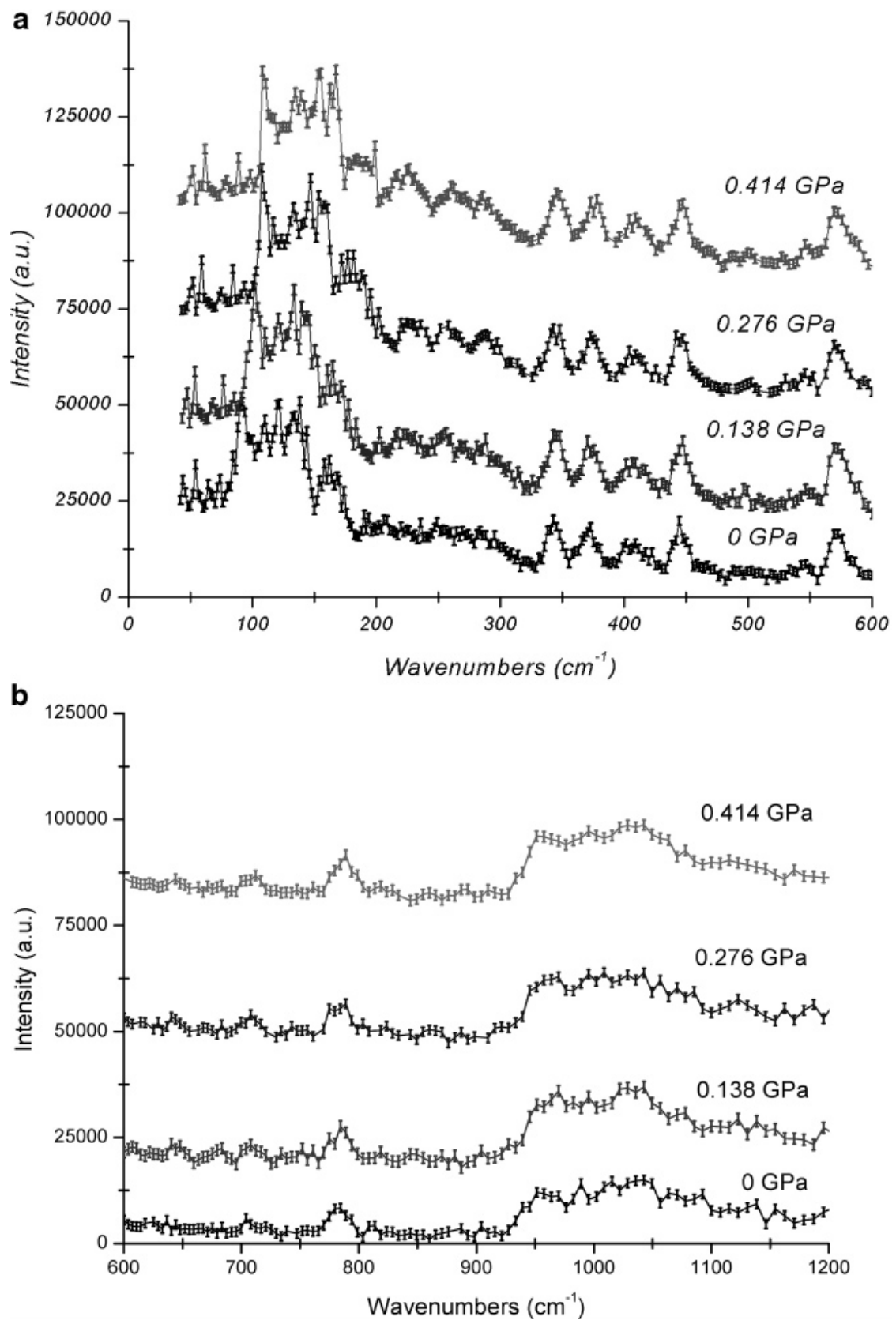

Figure 2. Experimental INS spectra of the TCNE-HMB complex collected on the FANS instrument at $50 \mathrm{~K}$ in the spectral ranges (a) $0-600$ $\mathrm{cm}^{-1}$ and (b) $600-1200 \mathrm{~cm}^{-1}$. Spectra are shown as a function of pressure and are offset for ease of comparison.

$0.41 \mathrm{GPa}$. This corresponds to a $5.37 \%$ decrease in the volume of the unit cell. As is shown in Table 1, the compression of the $b$ axis increased sharply between 0.138 and $0.276 \mathrm{GPa}$ in comparison to the $a$ and $c$ axis. The higher compression exhibited by the $b$ axis implies that the entire unit cell compresses anisotropically. This effect is not uncommon in layered materials, and in the TCNE-HMB complex the $b$ axis lies in the direction perpendicular to the stacked layers. These layers are held together only by weak van der Waals' interactions. Therefore, one would expect the $b$ direction of the unit cell to be much more compressible than either the $a$ or $c$ direction of the unit cell.

The calculated unit cell parameters as a function of pressure using the PBE and PW91 methods are summarized in Table 1. The observed errors for the experimental lattice parameters derived as a function of pressure are provided in the Supporting Information, Table S1. Comparison of the calculated unit cell parameters and the experimental parameters reveals large errors using both functionals. The RMS values at $0 \mathrm{GPa}(50 \mathrm{~K})$ are 0.333 (PBE) and 0.205 (PW91). The RMS values decreased slightly to 0.246 (PBE) and 0.167 (PW91) at $0.138 \mathrm{GPa}$. The RMS values systematically declined as the pressures increased, with values of 0.175 (PBE) and 0.124 (PW91), at $0.276 \mathrm{GPa}$. At $0.414 \mathrm{GPa}$, the calculated parameters were in close agreement with the experimental parameters and the RMS deviations are 0.026 (PBE) and 0.009 (PW91). The magnitude of the errors in the calculated cell vectors is clearly reflected in the RMS deviations between the experimental and calculated volumes. The PBE method yielded volume measurements with percent errors of $18.2 \%$ (0 GPa), $12.9 \%$ (0.138 GPa), 8.6\% (0.276 GPa) and $1.3 \%$ (0.414 GPa). Slightly smaller RMS deviations in the cell volume were obtained with the PW91 method. The RMS deviations were $11.3 \%$ (0 GPa), $8.9 \%$ (0.138 GPa), $1.3 \%$ (0.276 $\mathrm{GPa})$ and $0.3 \%(0.414 \mathrm{GPa})$ with the PW91 method.

It has been proposed that overestimation of lattice vectors arises from lack of proper van der Waals forces in current DFT 
TABLE 2: Experimental Peak and Theoretically Determined Vibrational Mode Energies of the TCNE-HMB Complex, Corresponding to Peaks Observed in the INS Spectra between 40 and $1200 \mathrm{~cm}^{-1}$ Collected under Both Ambient and Pressurized Conditions at $50 \mathrm{~K}$ Using the FANS Instrument ${ }^{a}$

\begin{tabular}{|c|c|c|c|c|c|c|c|c|c|}
\hline \multicolumn{2}{|c|}{$0 \mathrm{GPa}$} & \multicolumn{2}{|c|}{$0.138 \mathrm{GPa}$} & \multicolumn{2}{|c|}{$0.276 \mathrm{GPa}$} & \multicolumn{2}{|c|}{$0.414 \mathrm{GPa}$} & \multirow[b]{2}{*}{ mode no. ${ }^{b}$} & \multirow[b]{2}{*}{ molecular motion } \\
\hline INS & PW 91 & INS & PW 91 & INS & PW 91 & INS & PW 91 & & \\
\hline & 5 & & 7 & & 8 & & 12 & 1 & HMB-TCNE twisting \\
\hline & 30 & & 35 & & 40 & 51 & 52 & 2 & HMB - TCNE sliding \\
\hline 44 & 40 & 54 & 56 & 52 & 58 & 58 & 58 & 3 & HMB - TCNE stretching \\
\hline 54 & 60 & NR & 57 & 59 & 60 & 62 & 62 & 4 & HMB - TCNE tilting \\
\hline 64 & 64 & 67 & 67 & 75 & 73 & 78 & 75 & 5 & HMB-TCNE tilting \\
\hline 73 & 79 & 77 & 76 & 84 & 81 & 89 & 86 & 6 & HMB-TCNE tilting \\
\hline 85 & 82 & 96 & 96 & 94 & 99 & 97 & 99 & 7 & $\mathrm{HMB} \mathrm{CH}_{3}$ twist and TCNE $\mathrm{C}-\mathrm{C} \equiv \mathrm{N}$ out-of-plane torsion \\
\hline 91 & 93 & 102 & 104 & 107 & 108 & 108 & 112 & 8 & $\mathrm{HMB} \mathrm{CH}_{3}$ twist and TCNE C-C $\equiv \mathrm{N}$ out-of-plane torsion \\
\hline 99 & 101 & 110 & 109 & 116 & 117 & 116 & 125 & 9 & $\mathrm{HMB} \mathrm{CH}_{3}$ twist and ring deformation \\
\hline 108 & 110 & 127 & 126 & 133 & 135 & 135 & 131 & 10 & $\mathrm{C}-\mathrm{C} \equiv \mathrm{N}$ bend \\
\hline 121 & 120 & 134 & 133 & 142 & 145 & 141 & 146 & 11 & Methyl torsion \\
\hline 133 & 137 & 140 & 145 & 147 & 149 & 154 & 156 & 13 & $\mathrm{C}-\mathrm{C} \equiv \mathrm{N}$ bend \\
\hline 138 & 143 & 145 & 146 & 153 & 153 & NR & 157 & 14 & methyl torsion and $\mathrm{C}-\mathrm{C} \equiv \mathrm{N}$ in-plane torsion \\
\hline 145 & 150 & 151 & 147 & 158 & 163 & 161 & 161 & 15 & methyl torsion \\
\hline 155 & 154 & 156 & 157 & 167 & 168 & 167 & 168 & 16 & TCNE scissor mode \\
\hline 158 & 157 & 161 & 160 & 172 & 182 & 177 & 176 & 17 & methyl torsion \\
\hline 163 & 159 & 165 & 162 & 177 & 184 & 181 & 183 & 18 & $\mathrm{C} \equiv \mathrm{N}$ bend and $2 \mathrm{v}_{8}$ \\
\hline 169 & 168 & 172 & 173 & 181 & 185 & 190 & 192 & 19 & methyl torsion \\
\hline 174 & 170 & 177 & 178 & 188 & 186 & NR & 193 & 20 & methyl torsion \\
\hline 190 & 182 & 200 & 197 & 195 & 196 & 199 & 198 & 22 & ring deformation \\
\hline 193 & 193 & 203 & 200 & 202 & 204 & 205 & 205 & 23 & ring deformation \\
\hline 208 & 208 & 206 & 210 & 212 & 215 & 217 & 217 & 24 & $\mathrm{CH}_{3}$ torsion \\
\hline 220 & 218 & 221 & 222 & 224 & 227 & 226 & 226 & 25 & ring deformation and $\mathrm{CH}_{3}$ torsion \\
\hline 226 & 227 & NR & 228 & 228 & 230 & 230 & 231 & 26 & ring $-\mathrm{CH}_{3}$ bending out-of-plane \\
\hline 236 & 235 & 236 & 235 & 236 & 239 & $238(\mathrm{sh})$ & 237 & 27 & ring $-\mathrm{CH}_{3}$ bending out-of-plane \\
\hline 249 & 249 & 245 & 250 & $251(\mathrm{sh})$ & 253 & $251(\mathrm{sh})$ & 250 & 28 & $\mathrm{C}-\mathrm{C} \equiv \mathrm{N}$ bend \\
\hline 256 & 255 & 266 & 256 & 258 & 261 & 260 & 260 & 29 & ring $-\mathrm{CH}_{3}$ bending in-plane \\
\hline 269 & 267 & 271 & 263 & NR & 267 & 271 & 272 & 30 & ring $-\mathrm{CH}_{3}$ bending out-of-plane \\
\hline 284 & 282 & 281 & 279 & 284 & 282 & 286 & 284 & 31 & ring $-\mathrm{CH}_{3}$ bending in-plane \\
\hline 288 & 288 & 288 & 286 & 288 & 289 & 291 & 290 & 32 & ring $-\mathrm{CH}_{3}$ bending in-plane \\
\hline $302(\mathrm{sh})$ & 301 & 299 & 303 & NR & 295 & 306 & 304 & 33 & $\mathrm{C}-\mathrm{C} \equiv \mathrm{N}$ bend \\
\hline 314 & 313 & 314 & 317 & $314(\mathrm{sh})$ & 311 & 316 & 318 & 34 & $\mathrm{C}-\mathrm{C} \equiv \mathrm{N}$ bend \\
\hline 321 & 321 & 326 & 327 & 331 & 327 & $341(\mathrm{sh})$ & 340 & 36 & $\mathrm{C}-\mathrm{C}-\mathrm{C}$ rocking $(\mathrm{TCNE})$ \\
\hline 340 (sh) & 339 & $344(\mathrm{sh})$ & 341 & 343 & 340 & 347 & 347 & 35 & methyl torsion \\
\hline 343 & 344 & 347 & 347 & 352 & 350 & $352(\mathrm{sh})$ & 352 & 37 & methyl torsion \\
\hline 360 & 357 & $362(\mathrm{sh})$ & 359 & 363 & 364 & $365(\mathrm{sh})$ & 368 & 38 & $\mathrm{v}_{18}$ benzene-like mode Wilson scheme \\
\hline 367 (sh) & 366 & 368 & 363 & NR & 371 & 373 & 372 & 39 & $\mathrm{C}-\mathrm{C} \equiv \mathrm{N}$ bend \\
\hline 373 & 375 & 375 & 382 & 378 & 382 & 378 & 381 & 40 & $\mathrm{C}-\mathrm{C} \equiv \mathrm{N}$ bend \\
\hline $380(\mathrm{sh})$ & 378 & $396(\mathrm{sh})$ & 394 & 396 & 393 & $396(\mathrm{sh})$ & 395 & 41 & Methyl torsion \\
\hline 401 & 399 & 401 & 400 & 404 & 401 & 404 & 403 & 42 & methyl torsion \\
\hline 409 & 408 & 409 & 414 & 409 & 409 & 409 & 411 & 43 & out-of plane ring deformation \\
\hline 417 & 416 & $425(\mathrm{sh})$ & 424 & 425 & 425 & 428 & 428 & 44 & in-plane ring deformation \\
\hline 423 & 425 & 430 & 433 & 441 & 437 & NR & 441 & 45 & $\mathrm{C}-\mathrm{C}-\mathrm{C}$ rocking (TCNE) \\
\hline 444 & 446 & 447 & 448 & 447 & 446 & 447 & 449 & 46 & $\mathrm{C}-\mathrm{CH}_{3}$ deformation \\
\hline 467 & 466 & 469 & 467 & $473(\mathrm{sh})$ & 471 & 476 & 475 & 47 & out-of plane ring deformation \\
\hline 479 & 480 & 481 & 483 & 484 & 488 & 487 & 484 & 48 & in-plane ring deformation \\
\hline 487 & 486 & 487 & 489 & $490(\mathrm{sh})$ & 496 & 499 (sh) & 497 & 49 & $\mathrm{C}-\mathrm{C}-\mathrm{C}$ rocking (TCNE) \\
\hline 494 & 492 & 496 & 500 & 502 & 500 & NR & 510 & 50 & $\mathrm{C}-\mathrm{C}-\mathrm{C}$ wagging $(\mathrm{TCNE})$ \\
\hline 520 & 518 & 517 & 519 & NR & 521 & 517 & 518 & 51 & $\mathrm{C}-\mathrm{C}-\mathrm{C}$ bend \\
\hline 533 & 534 & 533 & 534 & 530 & 533 & 527 & 532 & 52 & $\mathrm{C}-\mathrm{C} \equiv \mathrm{N}$ bend \\
\hline 546 & 545 & 546 & 543 & 546 & 549 & 546 & 547 & 53 & $\mathrm{C}-\mathrm{C}-\mathrm{C}$ bending $(\mathrm{TCNE})$ \\
\hline 572 & 574 & 572 & 570 & 569 & 573 & 578 & 575 & 54 & $\mathrm{C}-\mathrm{C}-\mathrm{C}$ wagging $(\mathrm{TCNE})$ \\
\hline $586(\mathrm{sh})$ & 588 & 589 & 593 & 593 & 596 & $589(\mathrm{sh})$ & 583 & 55 & $\mathrm{C}-\mathrm{C}$ stretch (TCNE) \\
\hline 600 & 602 & 610 & 607 & 614 & 612 & 615 & 617 & 56 & $\mathrm{CH}_{3}$ out-of-plane deformation \\
\hline 637 & 639 & 640 & 646 & 640 & 646 & 644 & 649 & 57 & $v_{12}$ wilson benzene \\
\hline 667 & 665 & $667(\mathrm{sh})$ & 663 & 671 & 668 & 671 & 675 & 58 & $\mathrm{C} \equiv \mathrm{N}$ stretch \\
\hline 683 & 685 & 683 & 677 & 683 & 682 & 683 & 689 & 59 & $\mathrm{C} \equiv \mathrm{N}$ stretch \\
\hline 704 & 703 & 708 & 699 & 708 & 703 & 712 & 719 & 60 & $\mathrm{C} \equiv \mathrm{N}$ stretch \\
\hline 720 & 720 & 733 & 726 & 734 & 739 & 738 & 740 & 61 & ring breathing \\
\hline 738 & 736 & 742 & 741 & 742 & 750 & 747 & 755 & 62 & $\mathrm{C}-\mathrm{C}-\mathrm{C}$ wagging $(\mathrm{TCNE})$ \\
\hline 756 & 755 & 760 & 761 & 756 & 762 & 760 & 767 & 63 & $\mathrm{C}-\mathrm{C}$ stretch (TCNE) \\
\hline 774 (sh) & 771 & 774 & 769 & 775 & 778 & 779 (sh) & 782 & 64 & ring breathing \\
\hline 784 & 787 & 784 & 783 & 788 & 789 & 789 & 791 & 65 & $\mathrm{CH}_{3}$ out-of-plane deformation \\
\hline 810 & 812 & 818 & 814 & 823 & 829 & 829 & 827 & 66 & rocking $\mathrm{CH}_{3}$ in-plane \\
\hline 844 & 842 & 844 & 845 & 859 & 851 & 860 & 858 & 67 & rocking $\mathrm{CH}_{3}$ out-of-plane \\
\hline 856 & 855 & 870 & 865 & 871 & 872 & 876 & 878 & 68 & bending $\mathrm{CH}_{3}$ out-of-plane \\
\hline 876 (sh) & 878 & 882 & 884 & 887 & 891 & 893 & 895 & 69 & bending $\mathrm{CH}_{3}$ in-plane \\
\hline
\end{tabular}


TABLE 2 (Continued)

\begin{tabular}{|c|c|c|c|c|c|c|c|c|c|}
\hline \multicolumn{2}{|c|}{$0 \mathrm{GPa}$} & \multicolumn{2}{|c|}{$0.138 \mathrm{GPa}$} & \multicolumn{2}{|c|}{$0.276 \mathrm{GPa}$} & \multicolumn{2}{|c|}{$0.414 \mathrm{GPa}$} & \multirow[b]{2}{*}{ mode no. ${ }^{b}$} & \multirow[b]{2}{*}{ molecular motion } \\
\hline INS & PW 91 & INS & PW 91 & INS & PW 91 & INS & PW 91 & & \\
\hline 887 & 885 & 904 & 900 & 898 & 906 & 909 & 911 & 70 & $\mathrm{CH}_{3}$ out-of-plane deformation \\
\hline 916 & 915 & 927 & 927 & $930(\mathrm{sh})$ & 922 & 933 (sh) & 927 & 71 & bending $\mathrm{CH}_{3}$ in-plane \\
\hline 939 & 940 & 951 & 942 & 951 (sh) & 944 & NR & 957 & 72 & $\mathrm{C} \equiv \mathrm{N}$ stretching (TCNE) \\
\hline 970 & 971 & 970 & 966 & 970 & 974 & NR & 984 & 73 & $\mathrm{C}-\mathrm{CH}_{3}$ stretch and $\mathrm{CH}_{3}$ deformation \\
\hline 989 & 984 & 995 & 992 & 996 & 996 & 996 & 998 & 74 & $\mathrm{v}_{14}$ benzene-like mode Wilson scheme \\
\hline 1015 & 1015 & 1022 & 1025 & 1029 & 1030 & 1029 & 1031 & 75 & rocking $\mathrm{CH}_{3}$ out-of-plane \\
\hline 1042 & 1043 & 1043 & 1039 & 1043 & 1040 & 1043 & 1043 & 76 & rocking $\mathrm{CH}_{3}$ in-plane \\
\hline $1050(\mathrm{sh})$ & 1050 & $1056(\mathrm{sh})$ & 1051 & 1070 & 1067 & 1078 & 1079 & 77 & rocking $\mathrm{CH}_{3}$ in-plane \\
\hline 1065 & 1065 & 1078 & 1075 & 1085 & 1085 & 1100 & 1106 & 78 & $\mathrm{v}_{20}$ benzene-like mode Wilson scheme \\
\hline $1072(\mathrm{sh})$ & 1073 & 1085 & 1089 & 1107 (sh) & 1100 & 1110 & 1118 & 79 & bending $\mathrm{CH}_{3}$ out-of-plane \\
\hline 1155 & 1157 & 1178 & 1180 & 1187 & 1188 & NR & 1188 & 80 & $\mathrm{C} \equiv \mathrm{N}$ stretching \\
\hline
\end{tabular}

${ }^{a}$ The assignments of the molecular motions are similar to that previously reported. ${ }^{10}{ }^{b}$ Molecular modes are numbered according to ref 10 .
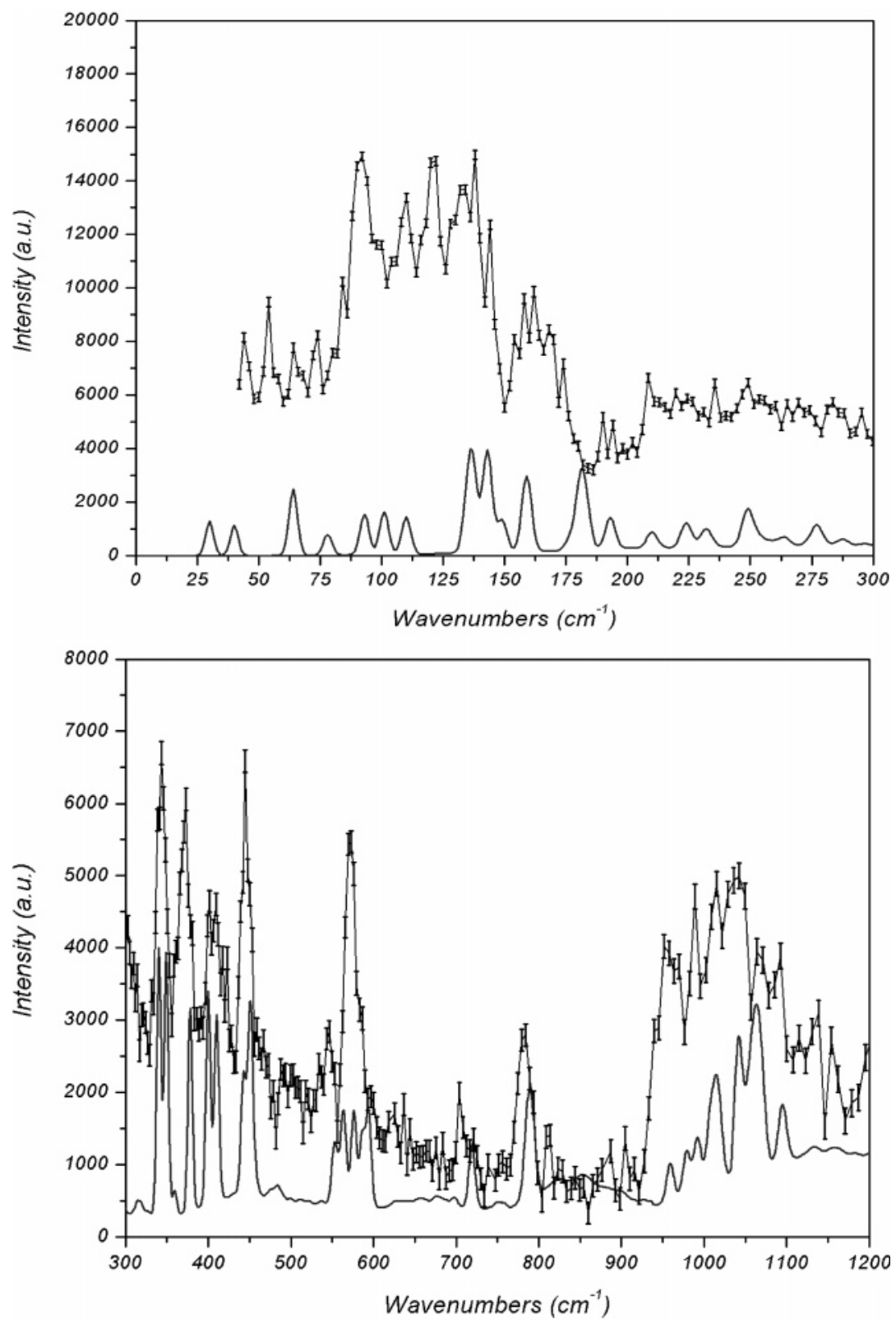

Figure 3. Comparison of the experimental INS spectrum at $50 \mathrm{~K}(0 \mathrm{GPa})$, shown with experimental error bars, and the calculated INS spectrum (solid line) shown in the (a) the $0-300 \mathrm{~cm}^{-1}$ and (b) $300-1200 \mathrm{~cm}^{-1}$ spectral ranges. Spectra are offset for ease of comparison.

functionals. ${ }^{46}$ In the case of the TCNE-HMB complex, the largest error in the individual lattice vectors is observed for the $b$ lattice parameter. Along the $b$ axis, as previously discussed, van der Waals forces are largely responsible for holding the crystal together. As pressure increases, the error decreases because the stacks are forced into a closer packed arrangement and the electron densities begin to overlap. Both the $a$ and $c$ lattice vectors systematically decrease as pressure increases. 

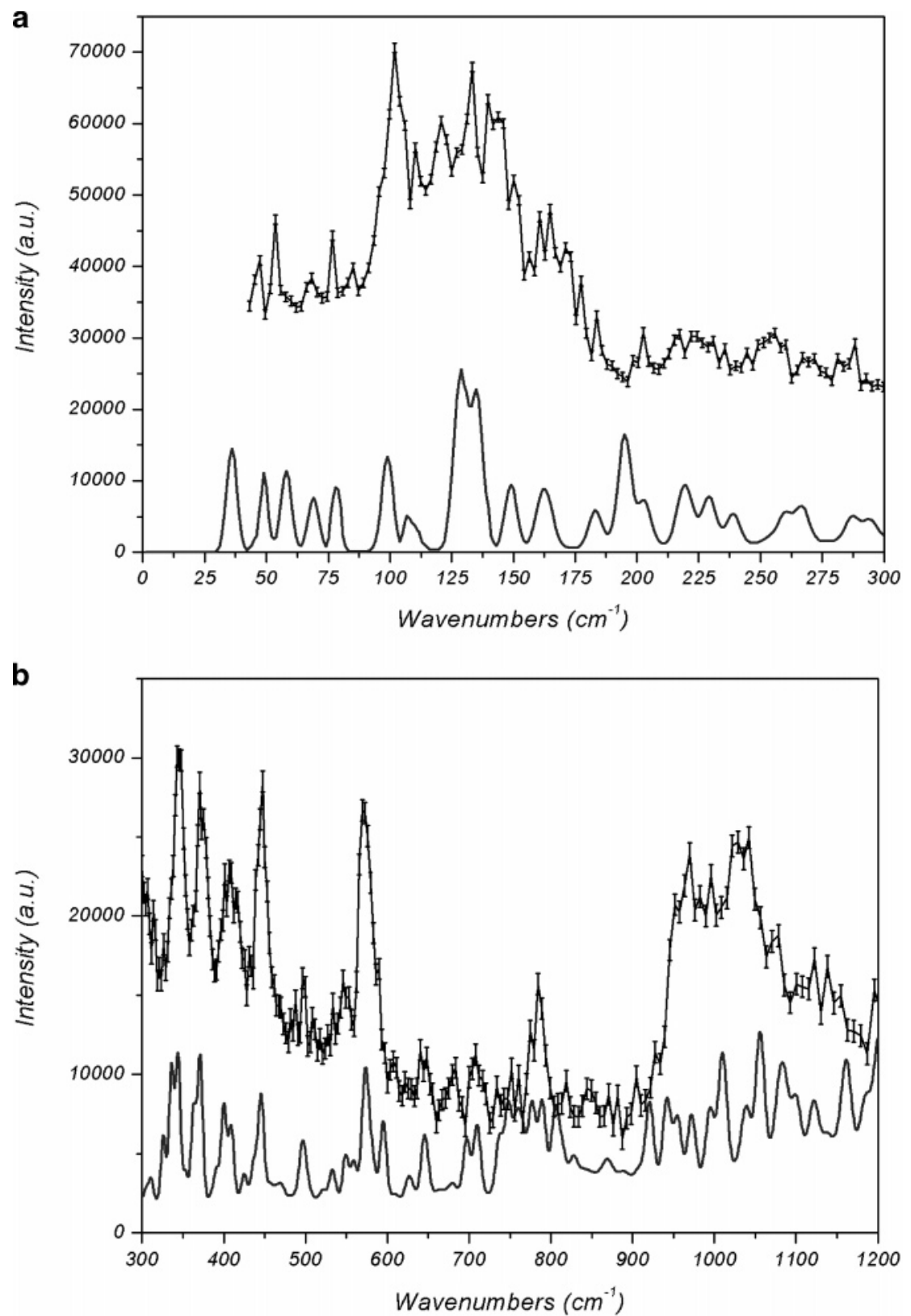

Figure 4. Comparison of the experimental INS spectrum at $0.138 \mathrm{GPa}$, shown with experimental error bars, and $50 \mathrm{~K}$ and the calculated INS spectrum (solid line) shown in the (a) $0-300 \mathrm{~cm}^{-1}$ and (b) $300-1200 \mathrm{~cm}^{-1}$ spectral regions using the PW91 functional. Spectra are offset for ease of comparison.

Although the calculated $b$ lattice vector slightly increases in length between 0 and $0.138 \mathrm{GPa}$, this correctly models the experimental behavior. Above $0.138 \mathrm{GPa}$, the length of the $b$ vector decreases. At $0.414 \mathrm{GPa}$, the values of all lattice parameters agree relatively well with experiment.

Molecular Vibrations. The inelastic neutron scattering spectra of the TCNE-HMB complex obtained at $50 \mathrm{~K}$ are shown in Figure 2 as a function of pressure. The spectra are offset for ease of comparison. At low frequency, in the spectral range of $40-300 \mathrm{~cm}^{-1}$, the INS spectra are characterized by several strong vibrational modes atop a broad envelope of intensity, which tails off to higher frequency. As is shown in the literature, this vibrational pattern is characteristic of hexamethylbenzene, which has several intense methyl torsions and phonon modes in this vibrational region. ${ }^{58}$

The center of the broad band at $0.414 \mathrm{GPa}$ is not noticeably shifted relative to that at $0 \mathrm{GPa}$, but it can be shown through close examination of Table 2, which details the vibrational assignments of the TCNE-HMB complex as a function of pressure, that several vibrational modes, in particular methyl torsions, have large vibrational shifts of $15 \mathrm{~cm}^{-1}$ or more. The two intense bands at 121 and $169 \mathrm{~cm}^{-1}$ in the $0 \mathrm{GPa}$ spectrum correspond to those at 141 and $190 \mathrm{~cm}^{-1}$ in the $0.414 \mathrm{GPa}$ spectrum, with vibrational shifts of 20 and $21 \mathrm{~cm}^{-1}$, respectively. Large red shifts have been suggested to result from of an increase in the ionic character of the EDA complex and the existence of a phase transition, termed the neutral-to-ionic transition, has been reported at higher pressures for several EDA complexes. ${ }^{59}$ In contrast to the large vibrational shifts at 120 and $169 \mathrm{~cm}^{-1}$, the two methyl torsion modes ca. $220 \mathrm{~cm}^{-1}(0$ $\mathrm{GPa})$ and $401 \mathrm{~cm}^{-1}(0 \mathrm{GPa})$, are only slightly affected by pressure application and vibrational shifts of only $3-6 \mathrm{~cm}^{-1}$ are observed. These relatively small shifts can likely be attributed to the intrinsic width of these vibrational bands in the INS spectra, as a result of overlapping of the fundamental 

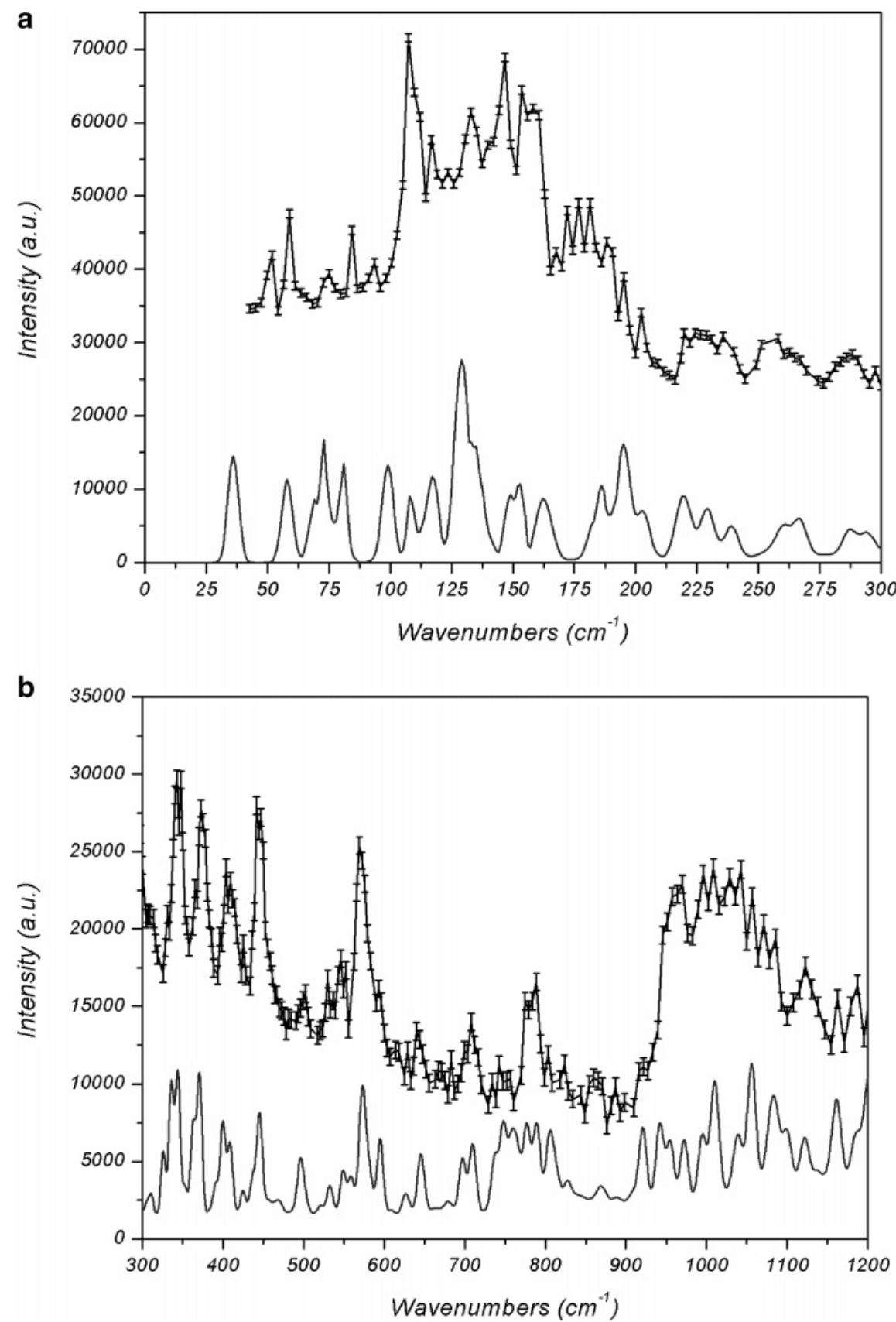

Figure 5. Comparison of the experimental INS spectrum at $0.276 \mathrm{GPa}$, shown with experimental error bars, and $50 \mathrm{~K}$ and the calculated INS spectrum (solid line) using the PW91 functional shown within the (a) $0-300 \mathrm{~cm}^{-1}$ and (b) $300-1200 \mathrm{~cm}^{-1}$ spectral ranges. Spectra are offset for ease of comparison.

vibrations with combination and overtone bands, leading to a smaller peak shift than is actually present.

Slight differences in the vibrational patterns as a function of pressure are also observed in the regions near 350, 375, and $440 \mathrm{~cm}^{-1}$. The relatively intense broad peak attributed to two methyl torsions ca. 340 and $343 \mathrm{~cm}^{-1}$ at $0 \mathrm{GPa}$ split into two distinct bands at a pressure of $0.276 \mathrm{GPa}$. The two methyl torsions at $373 \mathrm{~cm}^{-1}(0 \mathrm{GPa})$ and $380 \mathrm{~cm}^{-1}(0 \mathrm{GPa})$, which appear as a single strong band near $375 \mathrm{~cm}^{-1}$, also show splitting at 0.138 and $0.414 \mathrm{GPa}$. The evolution of the vibrational modes between $340-350$ and $370-380 \mathrm{~cm}^{-1}$ as a function of pressure is a direct result of the changing geometry of the methyl groups. As pressure increases, the staggered arrangement of the methyl groups, which is found at ambient conditions, becomes more pseudoplanar in nature. This, in turn, results in differing degrees of vibrational shifts. The vibration near $440 \mathrm{~cm}^{-1}$ can be attributed to a methyl deformation, which shows slight evidence of vibrational splitting at $0.276 \mathrm{GPa}$. Because no other vibrational modes are found in close proximity, factor group splitting could cause the splitting of this vibrational band, as symmetry related methyl groups are forced to reside in different environments as pressure increases.

DFT Calculations. The INS experimental frequencies and calculated vibrational frequencies were used to investigate the symmetries associated with the pressure-induced changes observed in the TCNE-HMB complex. Table 2 presents a comparison of the observed and calculated frequencies of the TCNE-HMB complex as a function of pressure. Descriptions of the molecular motion for the normal modes in the region studied are also presented in Table 2. These assignments of molecular motion are in close agreement with previously published data ${ }^{10}$ and the INS vibrational frequencies calculated 

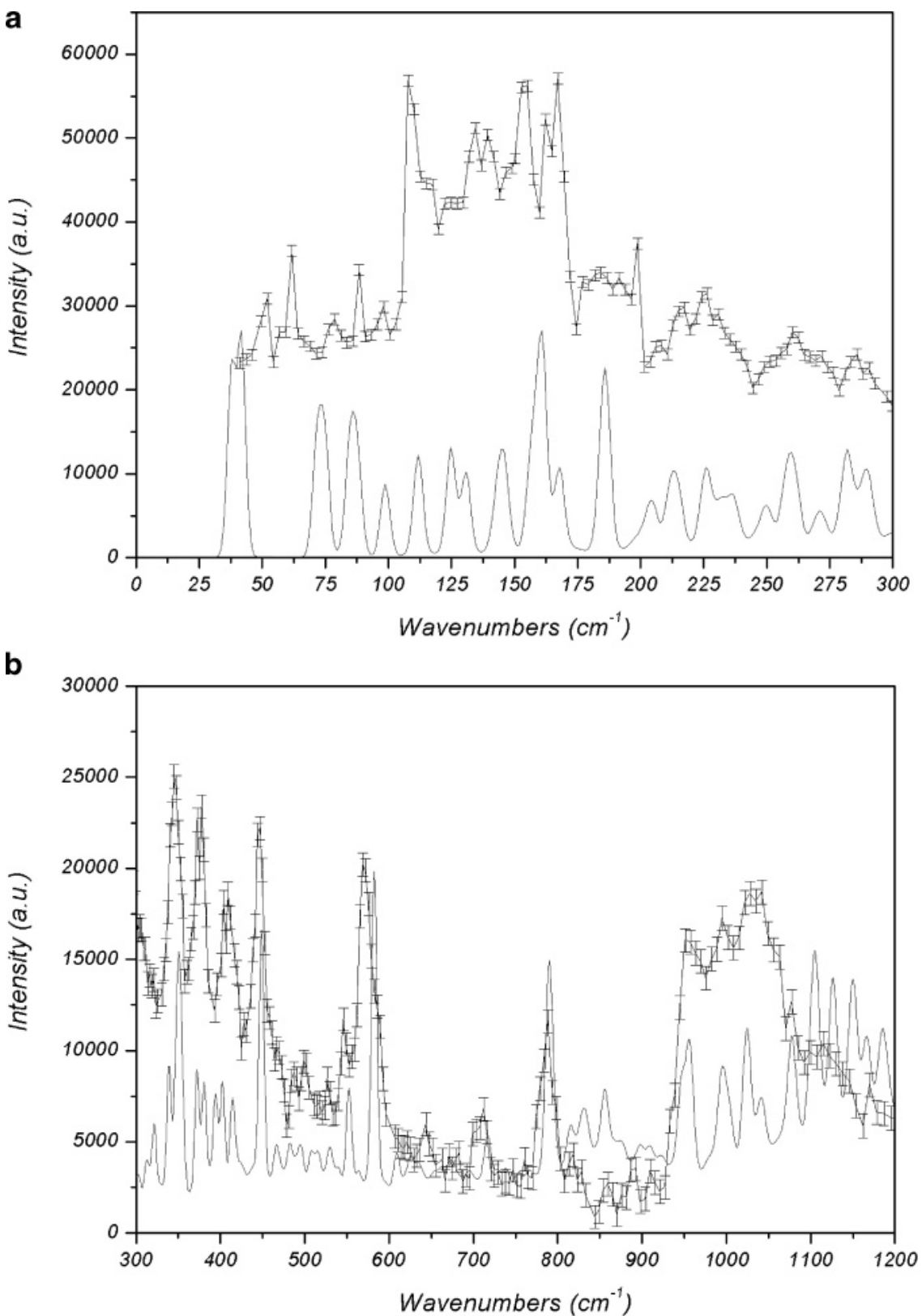

Figure 6. Comparison of the experimental INS spectrum, shown with experimental error bars, at $0.414 \mathrm{GPa}$ and $50 \mathrm{~K}$ and the calculated INS spectrum (solid line) in the (a) $0-300 \mathrm{~cm}^{-1}$ and (b) $300-1200 \mathrm{~cm}^{-1}$ spectral regions. Spectra are offset for ease of comparison.

with the PW91 functional closely model the experimental INS data for all pressures studied. At $0 \mathrm{GPa}$, the rms value is 2.41 $\mathrm{cm}^{-1}$. The corresponding rms value at $0.138 \mathrm{GPa}$ is $3.68 \mathrm{~cm}^{-1}$. The rms value increased slightly to 4.03 and $3.31 \mathrm{~cm}^{-1}$ at 0.276 and $0.414 \mathrm{GPa}$, respectively.

Individual comparisons of the experimental and calculated INS spectra are shown in Figures 3-6 as a function of pressure. A comparison of Figures 3 and 6 shows the intensities of the calculated INS spectra change significantly upon pressure increase, while the intensities of the experimental spectra remain virtually the same. This is particularly apparent in the spectral region between 800 and $900 \mathrm{~cm}^{-1}$, a region that is characterized by several out-of-plane motions of the methyl groups. Because the intensity of an INS transition is dependent upon hydrogen displacement, an increase in calculated intensity indicates the calculated molecular geometry is slightly different from the true crystal structure. However, this can only be confirmed through a high-resolution crystallographic analysis, which is beyond the scope of this paper.
A comparison of the calculated and experimental frequencies summarized in Table 2 and Figures 3-6 reveals, in general, good frequency and intensity agreement between calculation and experiment. The calculations presented in this paper are somewhat limited in the sense that they do not include vibrational intensity from the external lattice modes of the complex, which is reflected by the absence of strong phonon bands below $200 \mathrm{~cm}^{-1}$. To further improve upon the agreement of calculation and experiment, it will be necessary to perform a full crystal calculation on a "supercell".

Six intermolecular vibrational modes were predicted for each pressure. The HMB-TCNE stretching vibration was predicted at $40 \mathrm{~cm}^{-1}$ at $0 \mathrm{GPa}$ and was experimentally observed at 44 $\mathrm{cm}^{-1}$. Under pressure, the vibration experimentally red-shifted to $58 \mathrm{~cm}^{-1}$, with a calculated value of $58 \mathrm{~cm}^{-1}$. Three HMBTCNE tilting modes were experimentally observed at 54, 64, and $73 \mathrm{~cm}^{-1}$ at $0 \mathrm{GPa}$, with calculated counterparts of 60,64 , and $79 \mathrm{~cm}^{-1}$, respectively. These vibrations, at $0.414 \mathrm{GPa}$, shifted to 62,78 , and $89 \mathrm{~cm}^{-1}$ with calculated values of 62,75 , 
and $86 \mathrm{~cm}^{-1}$, respectively. The intermolecular modes of EDA complexes are highly coupled to the degree of electron transfer and such large red shifts indicate an increase in the ionic nature of the TCNE-HMB complex over this pressure range. ${ }^{60}$

Large vibrational shifts are also calculated to occur for three out-of-plane HMB vibrations near 842, 856, and $1073 \mathrm{~cm}^{-1}$. Confirmation of the shifts associated with these vibrations is not possible experimentally because of the low intensity. Several in-plane vibrations, which are calculated at $812,878,915$, and $1050 \mathrm{~cm}^{-1}$ have an average vibrational shift of $20 \mathrm{~cm}^{-1}$. Only the shift of the vibration at $1050 \mathrm{~cm}^{-1}$ can be confirmed experimentally.

\section{Conclusions}

Structural and molecular changes in the TCNE-HMB complex compressed to $0.417 \mathrm{GPa}$ were investigated using neutron diffraction, inelastic neutron scattering, and quantum chemical calculations. Analysis of the neutron powder diffraction at $50 \mathrm{~K}$ indicated anisotropic compression of the $b$ axis. The $b$ axis lies perpendicular to the molecular stacks, which only interact by van der Waals forces, making it highly compressible. It is expected that the spacing between the molecular stacks will continue to decrease until dissociation begins at approximately $6 \mathrm{GPa}^{44}$

The very good frequency agreement between the observed and computed inelastic neutron scattering spectra implies that the computational methods used provide a reasonably accurate quantitative description of the TCNE-HMB complex as a function of pressure. Several vibrations with a large degree of methyl motion had calculated vibrational shifts of greater than $10 \mathrm{~cm}^{-1}$ over the pressure range studied. Unfortunately, in some cases, we were unable to experimentally confirm the vibrational shifts predicted by the calculations, due to poor resolution or low vibrational intensity. Infrared or Raman spectroscopy with less spectral congestion can perhaps be used to identify these shifts.

Although some vibrational peaks were not able to be experimentally resolved, there seems to be good reason to suggest that these computations are reliable. To improve the agreement between experiment and calculation, it will be necessary to perform more extensive solid-state calculations with the use of a supercell. However, from the degree of frequency agreement between the experimental and simulated INS spectra, we can imply that the molecular geometry must be qualitatively correct. We suggest that the large degree of errors observed in the lattice vectors have little effect on the overall molecular structure, but a definite conclusion as to this will require confirmation of the molecular positions, via single-crystal diffraction, as a function of pressure.

Acknowledgment. The NIST Center for Neutron Research is acknowledged for providing neutron beam access on the FANS and BT-1 instruments. The Army Research Laboratory Major Shared Resource Center is thanked for access and support of the VASP program. An NRC Fellowship with the Army Research Laboratory supported J.C. during the course of this research.

Supporting Information Available: Table S1 reports the estimated error in the lattice vectors and volume of the TCNEHMB complex. This material is available free of charge via the Internet at http://pubs.acs.org.

\section{References and Notes}

(1) Miller, J. S.; Epstein, A. J. Molecule-based Magnets. In Crystal Engineering: From Molecules and Crystals to Materials; Braga, D., et al., Eds.; Kluwer: Dordrecht, The Netherlands, 1999; pp 43-53.
(2) Jerome, D.; Schultz, H. J. Organic conductors and superconductors. Adv. Phys. 2002, 51, 293.

(3) Singleton, J. J. Solid State Chem. 2002, 168, 689.

(4) Streetman, B.; Banarjee, S. Solid State Electronic Devices, 6th ed.; Prentice Hall: Englewood Cliffs, NJ, 2005.

(5) Freo, L. D.; Painelli, A.; Soos, Z. G. Phys. Rev. Lett. 2002, 89, $027402-1-4$

(6) Uno, B.; Okumura, N.; Seto, K. J. Phys. Chem. A 2000, 104, 3064.

(7) Tang, L.-T.; Wei, Y.; Wang, Y.; Hu, S.-W.; Liu, X.-Q.; Chu, T.W.; Wang, X.-Y. J. Mol. Struct. (THEOCHEM) 2004, 686, 25.

(8) Oisen, V.; Rabiller, P.; Katan, C. J. Phys. Chem. A 2004, 108, 11049

(9) Liao, M.-S.; Lu, Y.; Scheiner, S. J. Comput. Chem. 2002, 24, 624

(10) Ciezak, J. A.; Hudson, B. S. J. Mol. Struct. (THEOCHEM) 2005 $755,195$.

(11) Sawka-Dobrowolska, W.; Bator, G.; Sobczyk, L.; Pawlukojc, A.; Ptasiewicz-Bak, H.; Rundlöf, H.; Krawczyk, J.; Nowina-Konopka, M.; Jagielski, P.; Janik, J. A.; Prager, M.; Steinsvoll, O.; Grech, E.; NowickaScheibe, J. J. Chem. Phys. 2005, 123, 124305.

(12) Verdal, N.; Kozlowski, P. M.; Hudson, B. S. J. Phys. Chem A 2005, 109, 5724.

(13) Hudson, B. S.; Allis, D. G.; Parker, S. F.; Ramirez-Cuesta, R. J.; Herman, H.; Prinzbach, H. J. Phys. Chem. A 2005, 109, 3418.

(14) Ciezak, J. A.; Trevino, S. F. J. Mol. Struct. (THEOCHEM) 2005 , $723,241$.

(15) Plazanet, M.; Fontaine-Vive, F.; Gardner, K. H.; Forsyth, V. T.; Ivanov, A.; Ramirez-Cuesta, A. J.; Johnson, M. R. J. Am. Chem. Soc. 2005, 127, 6672.

(16) Eckert, J.; Sewell, T. D.; Kress, J. D.; Kober, E. M.; Wang, L. L.; Olah, G. J. Phys. Chem. A 2004, 108, 11369.

(17) Johnson, M. R.; Parlinski, K.; Natkaniec, I.; Hudson, B. S. Chem. Phys. 2003, 291, 53.

(18) Hermet, P.; Bantignies, J. L.; Rahmani, A.; Sauvajol, J. L.; Johnson, M. R. J. Phys. Chem. A 2005, 109, 4202.

(19) Hudson, B. S. J. Phys. Chem. A 2001, 105, 3949

(20) Saheki, M.; Yamada, H.; Yoshioka, H.; Nakatsu, K. Acta Crystallogr. 1976, B32, 662.

(21) Maverick, E.; Trueblood, K. N.; Bekoe, D. A. Acta Crystallogr. 1978, B34, 2777.

(22) Forster, R.; Kulevsky, N. J. Chem. Soc., Faraday Trans. 1 1973, 69, 1927.

(23) Rossi, M.; Buser, U.; Haselbach, E. Helv. Chim. Acta 1976, 59 , 1039 .

(24) Liptay, W.; Rehm, T.; Wehnig, D.; Schanne, W.; Baumann, W.; Lang, W. Z. Naturforsch. Teil A 1982, 37, 1427.

(25) Stanley, J.; Smith, D.; Latimer, B.; Devlin, J. P. J. Phys. Chem. 1966, 70, 0, 2011.

(26) Hall, B.; Devlin, J. P. J. Phys. Chem. 1966, 71, 465.

(27) Sakehi, M.; Yamada, H. Spectrochim. Acta 1976, A32, 1425.

(28) Rossi, M.; Haselbach, E. Helv. Chim. Acta 1979, 62, 140.

(29) Cesaro, S. N.; Martini, B.; Bencivenni, L.; Spoliti, M.; Maltese, M. Spectrochim. Acta 1980, A36, 165.

(30) Yamamoto, K.; Kabit, Md. H.; Hayashi, M.; Tominaga, K. Phys Chem. Chem. Phys. 2005, 7, 1945.

(31) Smith, M. L.; McHale, J. L. J. Phys. Chem. 1985, 89, 4002.

(32) McHale, J. L.; Merriam, M. J. J. Phys. Chem. 1989, 93, 526.

(33) Britt, B. M.; Lueck, H. B.; McHale, J. L. Chem. Phys. Lett. 1992, 190, 528 .

(34) Britt, B. M.; McHale, J. L.; Friedrich, D. M. J. Phys. Chem. 1995, 99, 6347.

(35) Kulinowski, K.; Gould, I. R.; Meyers, A. B. J. Phys. Chem. 1995 99, 9017.

(36) Britt, B. M.; McHale, J. L. Chem. Phys. Lett. 1997, 270, 551.

(37) Kulinowski, K.; Gould, I. R.; Ferris, N. S.; Meyers, A. B. J. Phys. Chem. 1995, 99, 17715 .

(38) Hayashi, M.; Yang, T.-S.; Yu, J.; Mebel, A.; Lin, S. H. J. Phys. Chem. A 1997, 101, 4156.

(39) Gott, J. R.; Maisch, W. G. J. Chem. Phys. 1963, 39, 2229.

(40) Offen, H. W.; Kadhim, A. H. J. Chem. Phys. 1966, 45, 269

(41) Nakayama, R.; Sasaki, M.; Osugi, A. J. Rev. Phys. Chem. Jpn. 1976, 46, 57 .

(42) Ewald, A. H. Trans. Faraday Soc. 1968, 64, 733

(43) Davis, K. M. C. In Molecular Association; Foster, R., Ed.; Academic: New York, 1975; Vol. 1, p 151.

(44) Jurgensen, C. W.; Peanasky, M. J.; Drickamer, H. G. J. Chem. Phys. 1985, 83, 6108.

(45) Yamada, H.; Saheki, M. Spectrochim. Acta 1980, 37A, 17.

(46) Byrd, E. F. C.; Scuseria, G. E.; Chabalowski, C. F. J. Phys. Chem B 2004, 108, 13100 .

(47) Details of the Harwood Engineering two-piston pressure intensifier system available for use at the NIST Center for Neutron Research can be found at http://users.ren.com/harwood.ma.ultranet/prim200k.html. 
(48) Specific instrumental details of the BT-1 powder diffractometer can be found at http://www.nenr.nist.gov/instruments/bt1.

(49) Larson, A. C.; VonDreele, R. B. General Structure Analysis System (GSAS); Los Alamos National Laboratory Report LAUR 86-748; LANL: Los Alamos, NM, 2000.

(50) Toby, B. H. J. Appl. Crystallogr. 2001, 34, 210.

(51) (a) Udovic, T. J.; Neumann, D. A.; Leão, J.; Brown, C. M. Nucl. Instrum. Methods Phys. Res. 2004, A517, 189. (b) www.ncnr.nist.gov/ instruments/fans.

(52) http://www.nenr.nist.gov/dave.

(53) Kresse, G.; Furthmuller, J. Vienna Ab Initio Simulation Package (VASP): The Guide; VASP Group, Institut fur Materialphysik, Universitat Wein, Sensengasse 8, A-1130 Wien, Vienna, Austria, 2003.

(54) Perdew, J. P.; Berke, K.; Ernzerhof, M. Phys. Rev. Lett. 1996, 77, 3865 .
(55) Perdew, J. P. In Electronic Structures of Solids '91; Ziesche, P., Eschrig, H., Eds.; Akademie-Verlag: Berlin, 1991.

(56) Vanderbuilt, D. Phys. Rev. B 1990, 41, 7892.

(57) Ramirez-Cuesta, A. J. Comput. Phys. Commun. 2004, 157, 226.

(58) (a) Stride, J. A.; Adams, J. M.; Johnson, M. R. Chem. Phys. 2005, 317, 143. (b) Krawczyk, J.; Mayer, J.; Natkaniec, I.; Nowina Konopka, M.; Pawkukojc, A.; Steinsvoll, O.; Janik, J. A. Physica B 2005, 362, 271.

(59) (a) Torrance, J. B.; Girlando, A.; Mayerle, J. J.; Crowley, J. I.; Lee, V. Y.; Batail, P.; LaPlaca, S. J. Phys. Rev. Lett. 1981, 47, 1747. (b) Torrance, J. B.; Vazquez, J. E.; Mayerle, J. J.; Lee, V. Y. Phys. Rev. Lett. 1981, 46, 253.

(60) Moreac, A.; Girard, A.; Delugeard, Y.; Marqueton, Y. J. Phys.: Condens. Matter 1996, 8, 3553. 